\title{
A formal graphical based process modeling methodology
}

\author{
Tormod Drengstig, Stein O. Wasbø and Bjarne A. Foss ${ }^{*}$
}

Department of Engineering Cybernetics, Norwegian University of Science and Technology, Trondheim, Norway.

\begin{abstract}
In this paper we present a representation scheme for chemical unit processes. The representation is based on a topological and a phenomenological abstraction of the process. The topological abstraction decomposes the process into control volumes and boundaries. The phenomenological abstraction represents the phenomena in the process using three general process characteristics, i.e. transport, reaction/generation and accumulation of mass and energy. For these entities we define a consistent set of graphical symbols that will be connected together in a network according to the modelers understanding of the process, giving a representation of the process. We further suggest to employ this representation in the development of a modeling methodology, where the symbols are related to differential and algebraic equations in order to represent a complete and consistent mathematical model. The methodology is successfully applied to two industrial processes, a ferromanganese furnace and an aluminum electrolysis cell. The latter will be used as an example. Simulations of the aluminum cell focusing on $\mathrm{AlF}_{3}$ dynamics are included.
\end{abstract}

\section{INTRODUCTION}

To develop a mathematical model of a chemical process, some sort of graphical sketch of the process is usually the first step. This graphical sketch is a conceptual picture of the process and it is used by the modeler when constructing the mathematical model. Several factors influence the chosen visualization of the process, e.g. 1) the properties that are believed to be important, 2) process assumptions like well mixed situation or equilibrium, 3 ) the complexity of the process, i.e. complex phenomena and reactions may be difficult to represent graphically, and hence, have to be represented in some kind of textual or mathematical terms, 4) the purpose of the model, e.g. is it to be used for control or design purposes, or 5) the model format, e.g. mechanistic vs. empirical.

In this work, we focus on defining a consistent and formal graphical representation of chemical unit processes. If such a formal representation can be defined, we foresee a development of a computer aided modeling tool able to interpret graphical symbols and guide the modeler towards a consistent mathematical model of her/his process. A representation solely based on detailed equations is not necessarily the best way to obtain efficient interaction in communicating with other resource personal with different modeling knowledge and background. Hence, we believe that a formalized graphical representation is a well suited means for such communication.

\section{MODELING METHODOLOGY}

The modeling methodology presented here is based on a formal graphical representation scheme. This scheme consists of two main parts, a topological and a phenomenological part. To the topological part belongs the decomposition of the process into modules representing control volumes (devices) having accumulation properties, and boundaries (connections) involving some kind of flow between devices. This is a similar approach as described in Marquardt (1994) and Perkins et al. (1994).
The phenomenological part describes the phenomena taking place inside the topological process components, e.g. chemical reaction or conductive heat flow. Hence, in this work we focus on the development of analytical or first principles mathematical models of lumped parameter systems.

\section{Topological process abstraction}

Topological process abstraction is the abstraction or decomposition of a system into a network of topology components, i.e. devices and connections, at several different abstraction levels. In order to separate the components at these different levels, we introduce composite and elementary components. Composite topology components are components containing a set of composite and/or elementary topology components, though, at the lowest level composed of elementary devices and connections. Only the elementary topology components contain a phenomenological description.

The basis for topological decomposition of plant processes is often guided by the physically separated unit processes constituting the plant. For the modularization of unit processes themselves, there is no similar approach. The basis may vary from chemical phase to temperature zone modularization within the same model, depending on the process and the scope of the model. The approach employed in this work is to choose chemical phase as a modularization basis. This means that e.g. a two-phase evaporator would be represented by one liquid and one gaseous elementary device in the model representation. The complete evaporator would be a composite device containing two elementary devices and one elementary connection.

The graphical symbols for elementary and composite components are given in Table 1. In order to connect the topology components into a complete network, we introduce various links or lines. These are given in Table 2.

*Author to whom correspondence should be addressed. E-mail: Bjarne.Foss@itk.ntnu.no 
The component connecting line is used between the topological components, and the relation element is used to indicate relations to higher levels of abstraction. In order to visualize the topological part of the model, a process topology diagram (PTD) is introduced.

Table 1: Symbols defined in the topological part of the graphical representation scheme.

\begin{tabular}{|c|c|c|}
\hline & elementary & composite \\
\hline device & $\begin{array}{l}\text { phase } \\
\text { source/sink } \\
\text { controller }\end{array}$ & \\
\hline connection & $\begin{array}{l}\text { phase } \\
\text { boundary } \\
\text { signal } \\
\text { connection }\end{array}$ & \\
\hline
\end{tabular}

Table 2: Relations for topological components.

\begin{tabular}{|ll|}
\hline \multicolumn{2}{|c|}{ relations } \\
relation \\
element \\
signal line \\
component \\
connecting line \\
\hline
\end{tabular}

In order to guide and support the modeler using these symbols, rules can be derived, e.g. a connection must be linked to at least two devices. More rules can be found in Drengstig et al. (1996).

We will exemplify the representation using a simple CSTR with one exotherm reaction. In the next section we will employ an aluminum electrolysis cell as a case study. A simple sketch of the CSTR is given in Fig. 1.

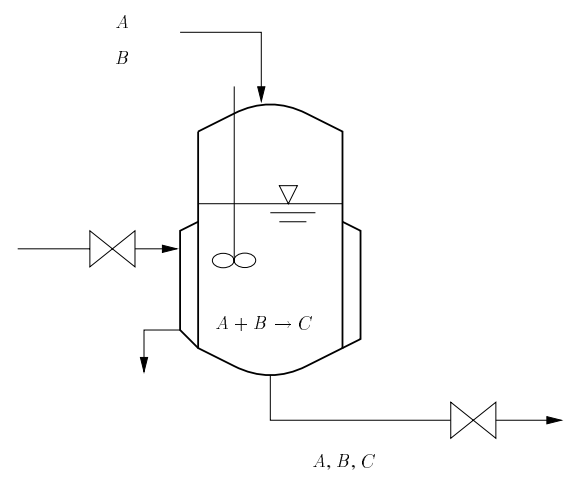

Figure 1: Sketch of a simple CSTR with one reaction.

For presentation purposes, controllers have been left out. The PTD for this unit process could be as in Fig. 2.

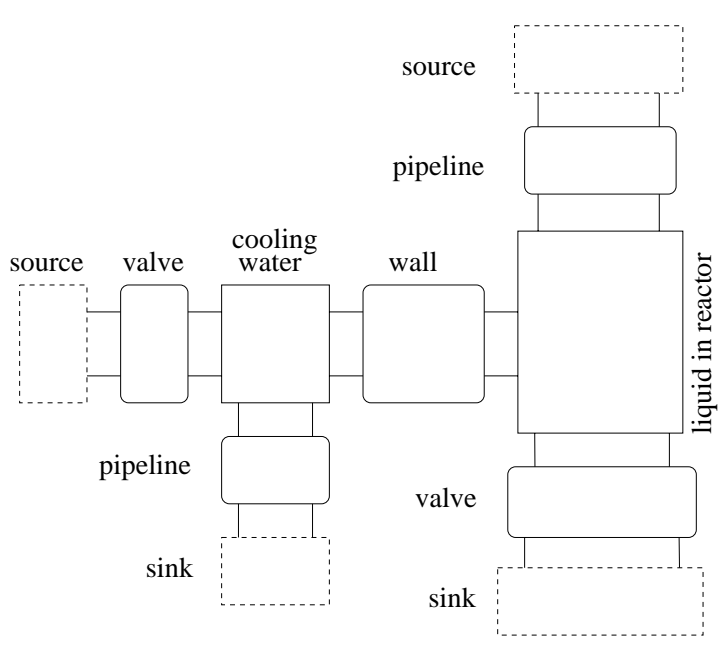

Figure 2: PTD of the CSTR in Fig. 1.

\section{Phenomenological process abstraction}

Phenomenological process abstraction is the abstraction of the behavior of elementary topological components into a network of phenomenological components. The identification of these components is based on three process characteristics, i.e. TRANSPORT, REACTION/GENERATION and ACCUMULATION. These characteristics are related to the following extensive quantities, mass and energy. The quantity mass is the mass of each chemical species. For these characteristics and quantities we have defined the symbols given in Table 3 .

Table 3: Symbols defined in the phenomenological part of the graphical representation scheme.

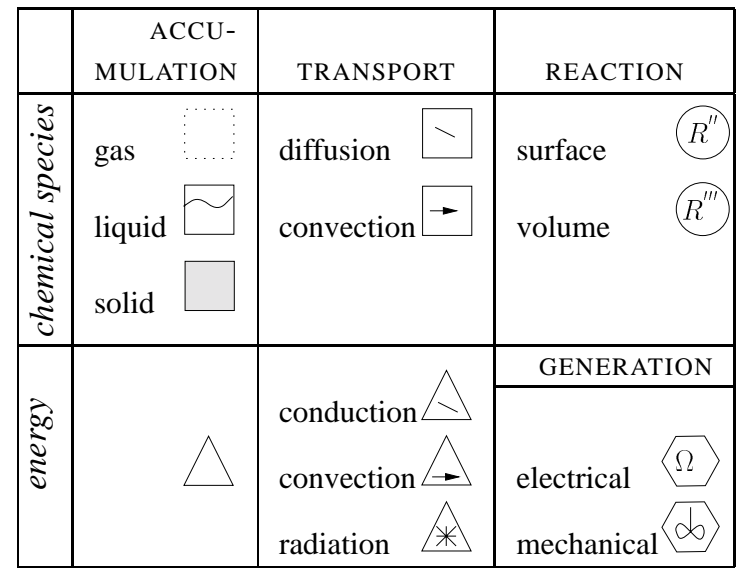

As for the topological components, we need relations as connecting elements between the phenomenological symbols. These are given in Table 4 . The direction of the arrows in Table 4 is not an indication of the direction of the flow, but rather it defines the positive direction for the flow. 
Table 4: Relations for phenomenological components.

\begin{tabular}{|l|}
\hline \multicolumn{2}{|c|}{ relations } \\
\hline material \\
flow \\
energy \\
flow \\
\hline
\end{tabular}

A transport, reaction and accumulation view (TRAV) is defined where the network of phenomenological symbols is constructed.

Employing these symbols and relations on the topological structure shown in Fig. 2, we get a combined PTD and TRAV representation as in Fig. 3.

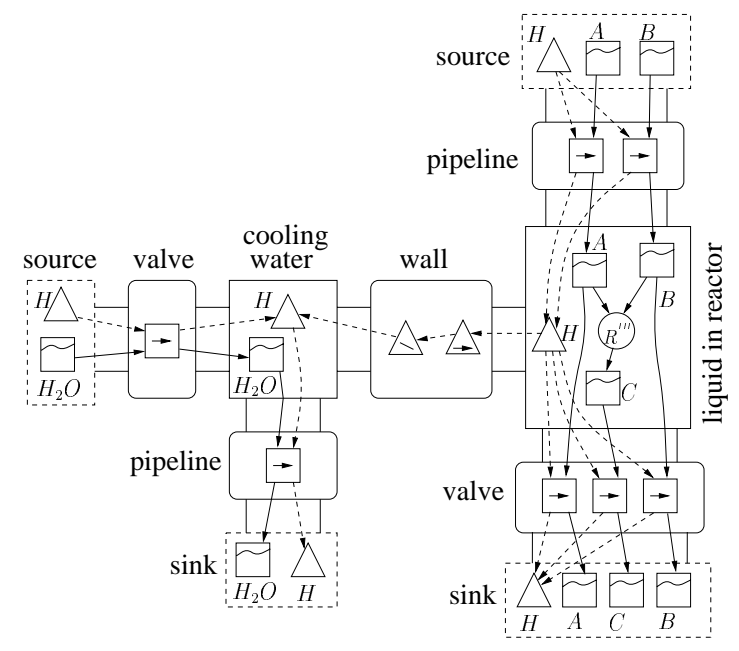

Figure 3: PTD and TRAV of the CSTR in Fig. 1.

Having defined this formal representation scheme for chemical processes, we now propose to use it as a basis for a modeling methodology and consequently a modeling tool. This implies that the representation of the process is a visualization of the mathematical model of the process. Hence, in order to achieve this mapping between the representation scheme and a consistent mathematical model, the symbols in the TRAV must be related to equations. For instance, in Table 3 we find the symbol for ACCUMULATION of chemical species. This symbol would be related to an ordinary differential equation (ODE) in the modeling methodology. Similarly, the TRANSPORT symbols would typically be related to algebraic equations (AE). The mapping from the representation scheme into equations have to be a one-to-one mapping in order to consistent. If we study the structure of a general balance equation, it can be written as

$$
\begin{array}{r}
\text { rate of change in holdup }=\text { rate of exchange } \\
+ \text { rate of generation/consumption }
\end{array}
$$

where the exchange term comprises all the inlet and outlet flows and the generation/consumption comprises the appearing or disappearing of chemical species or energy within the system boundaries. Viewing Eq. (1) together with the structure of symbols and relations in Fig. 3, we see that e.g. the chemical species symbol might have inputs, outputs and generation/consumption material flow lines attached to it, and hence, it maps the balance equation structure onto the representation scheme.

The symbols we have introduced and used in Fig. 3 indicate that our knowledge of the existence of a chemical species termed $A$ inside the reactor is represented by a square symbol. This does not imply that we consider species $A$ to be found in certain parts of the reactor. We know that $A$ physically can be found in the entire reactor volume, though we choose to represent its existence with a square symbol. Hence, the representation in Fig. 3 can be viewed upon as lumping the information of the amount of specie $A$ present in the reactor, to a symbol in the TRAV.

If we compare the representation of chemical species with our comprehension of chemical species being something of tangible matter, the use of the symbol in the TRAV should be intuitive. When it comes to the representation of a reaction, however, this is not at all a tangible matter as chemical species is. On the other hand, the reaction is taking place inside the reactor. The representation of the chemical reaction with a circular symbol, has two purposes. First it symbolically separates chemical species and reaction, and second, all the reactions occurring between $A$ and $B$ inside the control volume are visualized as taking place within the circular symbol. The representation is, however, not indicating the physical perspective, i.e. molecules colliding and generating new species inside the entire reactor, but rather the informational perspective, i.e. " $A$ and $B$ reacts to form $C$ ".

As for the topological decomposition, rules can be derived to support the modeling process. For instance, a set of chemical species can be included in a device, but only one energy symbol is allowed.

Experience shows that when the number of symbols inside each topological component exceeds a certain limit, the TRAV becomes difficult to read for the modeler. Further, if we study the mass flow symbols in Fig. 3, we find that each symbol carries redundant information in that wherever mass flows, energy flows. Moreover, these flows are linear dependent. This motivates a separation of the proposed representation into a mass based and an energy based structure, where the redundant information is hidden. This implies that the energy based TRAV represent the pure energy flow only, though the energy accumulation takes into account the energy related to mass flow. There is however no conflict with respect to equations and consistency using these separate representations.

For the CSTR, this new representation would then be given as in Figs. 4 and 5. The result of this separation, if we neglect the energy accumulations in the sources and sinks, is that the energy part could be represented by the cooling water, the wall and the liquid in reactor components only. This gives a reduction of insignificant information. 


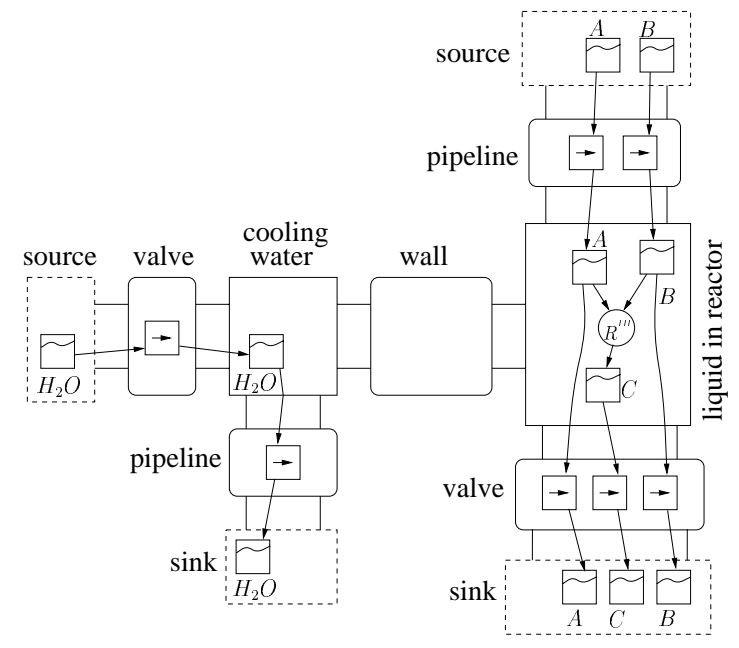

Figure 4: Mass based PTD and TRAV for the CSTR in Fig. 1.

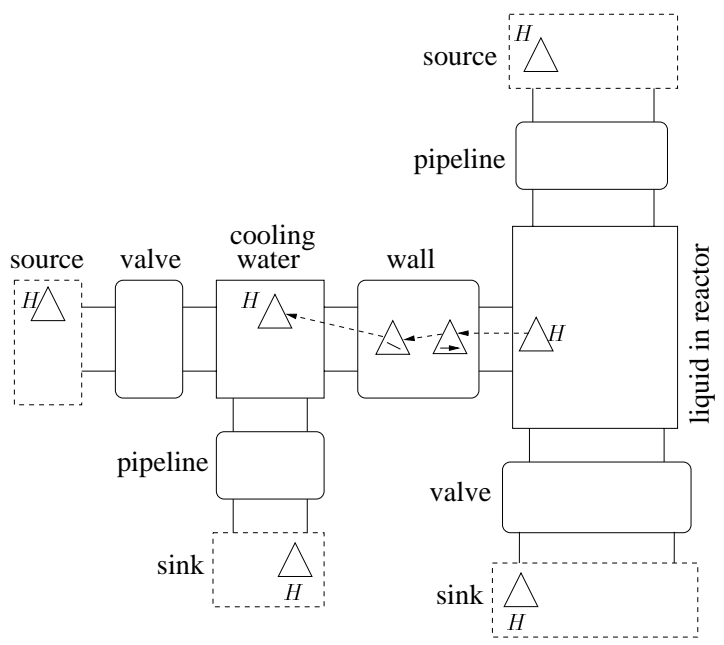

Figure 5: Energy based PTD and TRAV for the CSTR in Fig. 1.

\section{Modeling assumptions}

Generally, when decomposing a process into topological and phenomenological components, the modeler always simplify the physics of the process or make assumptions prior to the decomposition. The use of these simplifications and assumptions are often founded in the purpose of the model. This implies that there will always be a discrepancy between the real physics of the actual process and the PTD and TRAV representation. This again implies that, as the modeling process initiates, the modeler choose a level of simplification from where the process is decomposed. The resulting representation is a PTD and TRAV where further assumptions should be stated explicitly on this TRAV. Equilibrium is such an assumption, and hence, we introduce symbols for equilibrium modeling, given in Table 5 . However, this assumption should affect the mathematical equations, implying that the mapping from the PTD and TRAV is no longer consistent. In order to manipulate the underlying equations accordingly, an algorithm must interpret the PTD and TRAV and locate possible equilibrium assumptions. Such an algorithm is described in Drengstig et al. (1996), and its functionality follows the argumentation given in Ponton and Gawthrop (1991) for manipulating high index models into low index models.

Table 5: Equilibrium relations for phenomenological components.

\begin{tabular}{|l|}
\hline \multicolumn{1}{|c|}{ relations } \\
\hline phase- (material part) \\
and \\
reaction equilibrium \\
phase (thermal part) \\
equilibrium \\
\hline
\end{tabular}

The benefits of representing assumptions on the TRAV is that we keep the original decomposition of the process and, at the same time, enrich the information about the model. Other assumptions like e.g. constant volume also impose constraints on the model equations. The representation of these assumptions and the according manipulation of equations is a topic for present research.

An example of how the initial level of simplification described above will affect the PTD structure is given in the next case study.

There are presently two implementations of the modeling methodology, one in SIMULINK (The MathWorks 1992) and one in $\mathrm{C}++$.

\section{CASE STUDY}

We will here exemplify the use of the methodology on an aluminum electrolysis cell. A sketch of the process is given in Fig. 6. The main feed, $\mathrm{Al}_{2} \mathrm{O}_{3}$, is dissolved in the bath and electrically reduced to aluminum according to following reaction

$$
2 \mathrm{Al}_{2} \mathrm{O}_{3}+3 \mathrm{C} \rightarrow 4 \mathrm{Al}+3 \mathrm{CO}_{2}
$$

The compound $\mathrm{AlF}_{3}$ is added to reduce the melting point of the bath. However, the observed cell behavior after adding $\mathrm{AlF}_{3}$ is not fully understood. Hence, the aim of our work is primarily to develop a model of the $\mathrm{AlF}_{3}$ dynamics, and secondly, to use it for model based control.

Some typical process characteristics are the current through the cell which is typically in the range of 150$200 \mathrm{kA}$. The voltage across each cell is approximately $4 \mathrm{~V}$. The energy consumption of producing $1 \mathrm{~kg}$ of aluminum ranges from 13 to $15 \mathrm{kWh}$. One cell produces around 1000 - $1500 \mathrm{~kg}$ aluminum pr. day, and up to 200 cells are coupled in series in large plant halls, giving a total voltage drop of approximately $1000 \mathrm{~V}$ over the cell lines. 


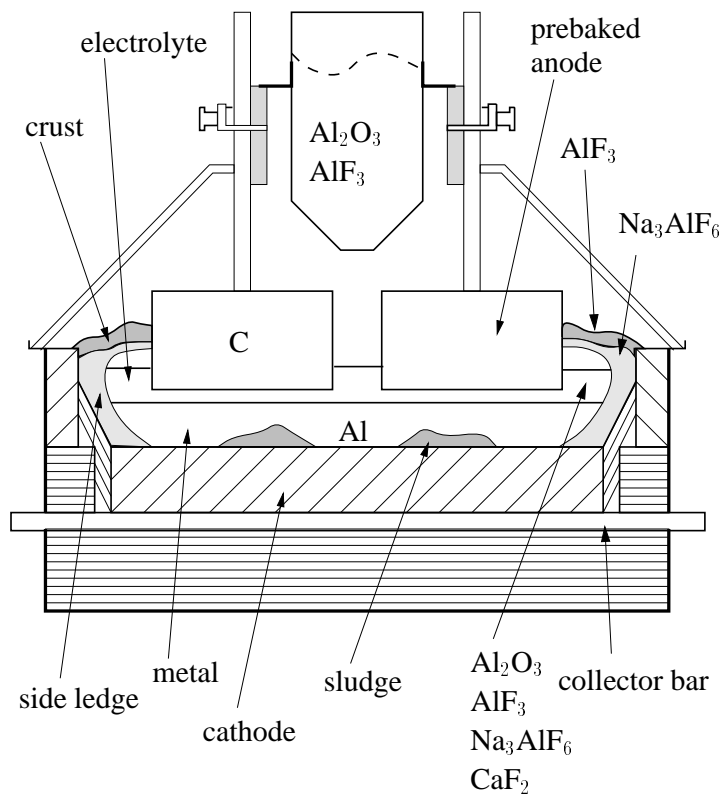

Figure 6: Sketch of an aluminum electrolysis cell.

As mentioned earlier, we have separated the PTD and TRAV into a mass and an energy aspect. For the aluminum cell, this approach become very useful in that it demonstrates that the mass and energy aspect become different since they focus on different phenomena of the process. The mass and energy aspects of the aluminum cell are given in Figs. 7 and 8.

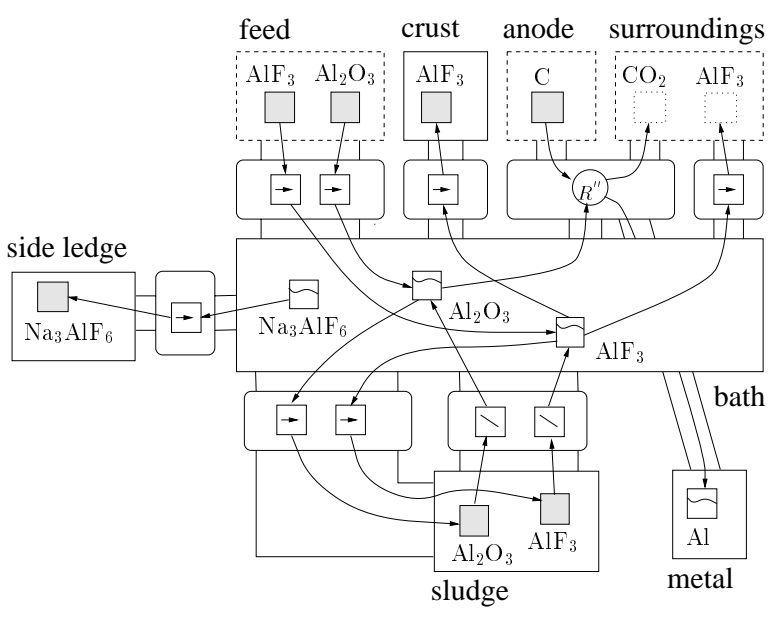

Figure 7: PTD and TRAV for the aluminum electrolysis cell, mass aspect.

Wherever current passes through an electrolyte and some kind of reaction occurs, there is always a reduction and an oxidation reaction occurring at the cathode and the anode respectively. This implies that inside the bath there is a transport of charged ions between the cathode and anode. In making a dynamic model of the cell, it is often satisfactory to model the overall reaction, i.e. the sum of both reactions. This is done in the representation in Fig. 7, and it exemplifies the level of assumption and simplification we chose prior to the modularization. This assumption implies that a surface reaction has to be moved from one surface to another, in this case from the cathode to the anode, and that the surface (connection) between bath and metal is neglected in the mass based topological decomposition. It is however interesting to note that the connection between bath and metal is used when modeling the heat flow in the energy based topological decomposition given in Fig. 8.

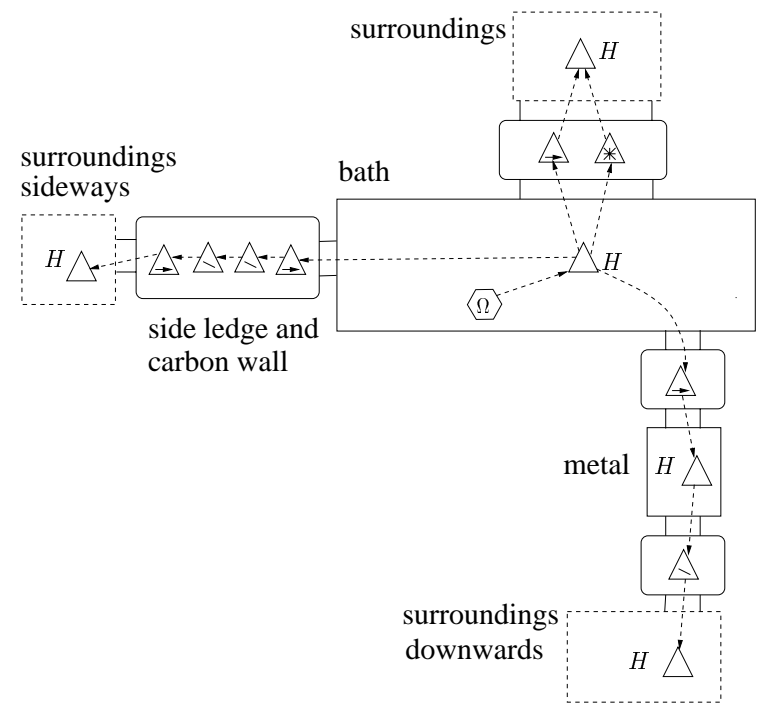

Figure 8: PTD and TRAV for the aluminum electrolysis cell, energy aspect.

Another interesting effect that can be observed is that due to the solid aggregate state of the side ledge, we do not model the energy accumulation, but rather assume a heat flow through the side ledge. There is however an accumulation of $\mathrm{Na}_{3} \mathrm{AlF}_{6}$ in the side ledge, see Fig. 7, implying that from a mass point of view, we consider the side ledge a device, but from an energy point of view, the side ledge is a connection. Hence, mathematically there will be a dependency between the amount of side ledge and to the conductive heat flow.

\section{MODEL VALIDATION}

As mentioned, we focus on the $\mathrm{AlF}_{3}$ dynamics of the aluminum cell. The model presented in Figs. 7 and 8 has been validated against measurements of $\mathrm{AlF}_{3}$ consentration, also termed acidity, and bath temperature. The main result of this validation is that there are dynamics the model structure does not capture (Drengstig et al. 1997). Based on this experience, we use measurements of acidity to estimate $\mathrm{AlF}_{3}$ disturbances and bath temperature measurements to estimate energy disturbances. The result of these estimations are shown in Figs. 9 and 10, respectively. Since the $\mathrm{AlF}_{3}$ and energy balances are exposed to several different disturbances, the estimated disturbances are the equivalent disturbances, i.e. we do not address the source, but rather the level of the disturbances.

Due to the estimated equivalent $\mathrm{AlF}_{3}$ disturbance, the acidity shows good conformity in Fig.9. However, the effect on the bath temperature is rather poor. 

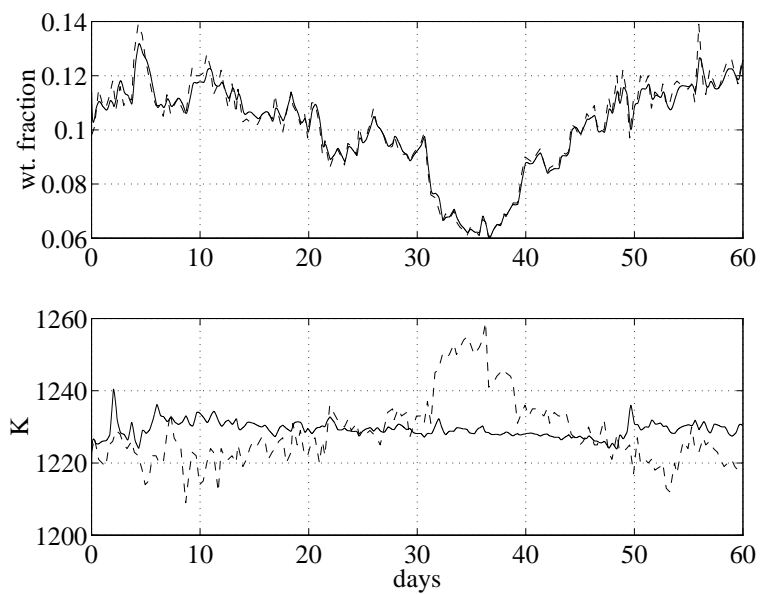

Figure 9: Above: Measured (dashed) and simulated (solid) acidity. Below: Measured (dashed) and simulated (solid) bath temperature.

In Fig. 10, the bath temperature shows good conformity due to the estimated equivalent energy disturbance. Moreover, the main variations in the acidity response also shows good conformity with the measurements.
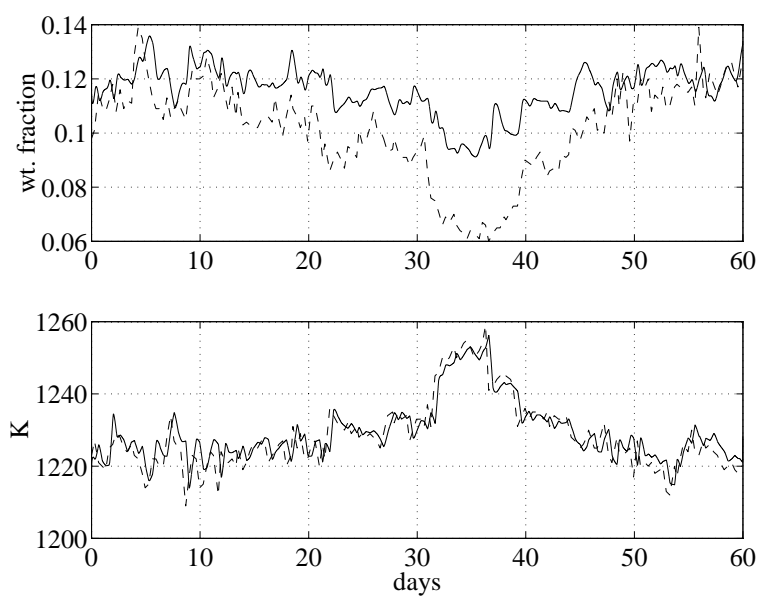

Figure 10: Above: Measured (dashed) and simulated (solid) acidity. Below: Measured (dashed) and simulated (solid) bath temperature.

Further, to obtain the result in Fig. 9, the level of the estimated equivalent $\mathrm{AlF}_{3}$ disturbance is $\pm 100 \%$ of the registred $\mathrm{AlF}_{3}$ input, which is too large to be reasonable. On the other hand, the level of the estimated equivalent energy disturbance is only $\pm 10 \%$ of the average heat loss from bath to surroundings (Drengstig et al. 1997). This indicates that the $\mathrm{AlF}_{3}$ dynamics is dominated by energy disturbances rather than $\mathrm{AlF}_{3}$ disturbances. Hence, further modeling effort should focus on improving the energy balance.

\section{DISCUSSION}

The modeling methodology presented in this paper is based on a formal representation scheme for chemical unit processes. The methodology facilitates easy model development and enlargement and it supports communication between resource personal with different background. However, experience shows that the representation scheme may become crowded if the number of chemical species and/or reactions are high. Hence, a separation of the representation into a mass based and an energy based representation has been advantageous. The main application of the methodology is to model lumped parameter systems using extensive quantities, e.g. number of moles, rather then intensive, e.g. concentration. Equilibrium modeling and index problems are also addressed. Based on the topological and phenomenological representation it is possible to develop modeling rules and thereby achieve consistent modeling. The methodology is applied to a ferro manganese furnace (Wasbø 1996) and an aluminum electrolysis cell.

Suggestions for future work are on representing and handling assumptions and constraints in general, to develop new and improved symbols and to implement the methodology in a more suitable environment, e.g. Visual C++.

\section{ACKNOWLEDGMENT}

This work was financially sponsored by Hydro Aluminium AS and the Research Council of Norway through the Expomat and Prosmat programs. The authors would also like to thank Associate Professor Dag Ljungquist for fruitful discussions.

\section{REFERENCES}

Drengstig, T., D. Ljungquist and B. A. Foss (1997). A study on the $\mathrm{AlF}_{3}$ and temperature dynamics of an aluminum electrolysis cell. In: Literature for the 7th Nordic Process Control Workshop. Wadahl, Norway. pp. 276-284.

Drengstig, T., S. O. Wasb $\varnothing$ and B. A. Foss (1996). A formal graphical based process modeling methodology. Technical Report 96-41-W. Department of Engineering Cybernetics, Norwegian University of Science and Technology, Trondheim, Norway.

Marquardt, W. (1994). Trends in computer-aided process modeling. In: Proc. of PSE'94. Kyongju, Korea. pp. 1-24.

Perkins, J. D., R. W. H. Sargent and R. Vázquez-Román (1994). Computer generation of process models. In: Proc. of PSE'94. Kyongju, Korea. pp. 123-125.

Ponton, J. W. and P. J. Gawthrop (1991). Systematic construction of dynamic models for phase equilibrium processes. Comput. chem. Engng. 15(12), 803-808.

The MathWorks, Inc. (1992). Simulink, user's and reference guide.

Wasbø, Stein O. (1996). Ferromanganese Furnace Modeling using Object Oriented Principles. Dr.ing. thesis. Department of Engineering Cybernetics, Norwegian University of Science and Technology. Trondheim, Norway. 